\title{
Study of the Safety, Immunogenicity and Efficacy of Attenuated and Killed Leishmania (Leishmania) major Vaccines in a Rhesus Monkey (Macaca mulatta) Model of the Human Disease
}

\author{
VF Amaral, A Teva, MP Oliveira-Neto*, AJ Silva, MS Pereira, E Cupolillo, R Porrozzi, \\ SG Coutinho, C Pirmez**, SM Beverley***, G Grimaldi Jr/+
}

Departamento de Imunologia **Departamento de Bioquímica e Biologia Celular, Instituto Oswaldo Cruz-Fiocruz *Centro de Pesquisa Hospital Evandro Chagas-Fiocruz, Av. Brasil 4365, 21045-900 Rio de Janeiro, RJ, Brasil ***Department of Molecular Microbiology, Washington University Medical School, St Louis, MO, USA

We have compared the efficacy of two Leishmania (Leishmania) major vaccines, one genetically attenuated (DHFR-TS deficient organisms), the other inactivated [autoclaved promastigotes (ALM) with bacillus CalmeteGuérin (BCG)], in protecting rhesus macaques (Macaca mulatta) against infection with virulent $\mathrm{L}$. (L.) major. Positive antigen-specific recall proliferative response was observed in vaccinees $(79 \%$ in attenuated parasite-vaccinated monkeys, versus 75\% in ALM-plus-BCG-vaccinated animals), although none of these animals exhibited either augmented in vitro gamma interferon (IFN- $\gamma$ ) production or positive delayed-type hypersensitivity $(D T H)$ response to the leishmanin skin test prior to the challenge. Following challenge, there were significant differences in blastogenic responses $(p<0.05)$ between attenuated-vaccinated monkeys and naïve controls. In both vaccinated groups very low levels of antibody were found before challenge, which increased after infective challenge. Protective immunity did not follow vaccination, in that monkeys exhibited skin lesion at the site of challenge in all the groups. The most striking result was the lack of pathogenicity of the attenuated parasite, which persisted in infected animals for up to three months, but were incapable of causing disease under the conditions employed. We concluded that both vaccine protocols used in this study are safe in primates, but require further improvement for vaccine application.

Key words: Leishmania (L.) major - rhesus macaques (Macaca mulatta) - vaccination - attenuated and killed promastigotes bacillus Calmete-Guerin (BCG)

Leishmaniasis is one of the major parasitic diseases targeted by the World Health Organization (WHO). There are an estimated 12 million cases worldwide. Two million new cases occur each year and 350 million people are at risk of infection (WHO/CID/Leish/98.9 Add.1). Control of leishmaniasis in several (sub)tropical areas is complicated by the variety of different Leishmania species and their diverse clinical manifestations and by the fact that each parasite species has a unique epidemiologic pattern. Until effective vaccines are available, environmental-oriented control measures (such as vector and reservoir control and epidemiologic surveillance) and chemotherapy of leishmaniasis (based on the use of leishmanicidal drugs) will continue to be the best options for prevention and containment of the disease. Current problems are (i) the

This work was supported by grants from the UNDP/World Bank/WHO Special Program for Research and Training in Tropical Diseases, from Fiocruz and from Faperj.

The data presented in this paper have been submitted by VF Amaral as part of a thesis for a $\mathrm{PhD}$ degree in Cellular and Molecular Biology at Fiocruz (Rio de Janeiro, Brazil).

${ }^{+}$Corresponding author. Fax: +55-21-2280.1589. E-mail: grimaldi@gene.dbbm.fiocruz.br

Received 9 January 2002

Accepted 7 June 2002 fact that sustained vector control utilizing large scale insecticide spraying (Dye 1996) in developing countries is costly and not feasible; (ii) Leishmania spp. readily acquires resistance to antimonial drugs (which despite their toxic properties still remain the treatment of choice); and (iii) response to treatment varies considerable depending on the parasite species involved and the clinical form or stage of the disease (reviewed by Grimaldi \& Tesh 1993).

Leishmania (L.) major is the causative agent of zoonotic cutaneous leishmaniasis (CL) and affects millions of people in many parts of the world (WHO/CID/ Leish/98.9 Add.1). Clinical studies have shown that healing of CL and immunity in $L$. (L.) major-infected patients are correlated with increased production of IFN- $\gamma$ and positive leishmanin skin test (LST) response (Kemp et al. 1994). Following convalescence to CL or the resolution of an induced skin lesion by artificial infection (called 'leishmanization'), individuals are protected from subsequent natural or experimental infection (Guirges 1971). These data have suggested that vaccination may prove to be the most cost-effective intervention method for the prevention of clinical disease. However, preventive vaccination with live parasites produces lesions in a small proportion of the vaccinees that requires medical treatment (Naggan et al. 1972). As a consequence, this type of immunization represents serious limitations.

Despite several efforts towards vaccine development, no effective recombinant vaccine currently exists in humans (reviewed by Grimaldi 1995). The induction of 
protective immunity using either inactivated or attenuated live vaccines would be a significant step in the control of the disease. The use of nonliving Leishmania vaccines against New World CL is only in experimental stages. The first field trial in Brazil evaluating the efficacy of a polyvalent vaccine showed that more than $70 \%$ of the vaccinees became LST positive, but the study was inconclusive by low incidence rate in the control group (Mayrink et al. 1979). In two placebo-controlled randomized trials in the Amazon region of Brazil the results showed $23 \%$ and $60 \%$ protection, respectively, but each study failed to reach statistical significance (Antunes et al. 1986). In a more recent human leishmanial vaccine trial in Equador (Armijos et al. 1998) the protective efficacy obtained (of $73 \%$ at one year) indicates this may be a promising approach. In contrast, a vaccine consisting of a single dose of whole-cell ALM mixed with BCG was recently shown to be ineffective in a controlled large scale human trial in Iran. The preferential protective efficacy (of $18 \%$ and $78 \%$ for the first and second years, respectively) observed in boys during the study period may have resulted from a greater booster effect produced by repeated exposure to infected sand flies (Sharifi et al. 1998). In a second trial (Momeni et al. 1999), LST conversion was significantly greater in vaccinees than in the BCG group (36.2\% vs $7.9 \%$ on day- 80 and $33 \%$ vs $18.5 \%$ after one year, respectively), but cumulative incidence rates for 2 years were similar in both groups (18\% vs $18.5 \%$ ). These observations indicate therefore that there is still much to be done for assessing the effectiveness of vaccination in the absence of natural challenge.

Nonhuman primates appear to have significant advantages over conventional laboratory animals in terms of modeling CL for purposes of vaccine evaluation. The Asian rhesus macaques (M. mulatta) are quite susceptible to leishmanial infection, develop a human-like disease (namely, the self-healing CL and resistance to homologous challenges), exhibit antigen-induced $\mathrm{T}$ lymphocyte reactivity both in vivo and in vitro (Amaral et al. 1996, 2001), and can be protected quite effectively by Leishmania vaccination (Kenney et al. 1999, CamposNeto et al. 2001). The present study was aimed to compare the safety and protective potential of two $L$. (L.) major vaccines (one attenuated parasites, the other heat-killed promastigotes + BCG) in the rhesus monkey model of the disease. The induction of parasite-specific immune responses was assessed following vaccination and challenge infection with virulent $L$. (L.) major.

\section{MATERIALS AND METHODS}

Animals - Primates used in this study were laboratorybred and -reared young adult (3- to 10-year-old, weighing between 4,360 and $14,420 \mathrm{~g}$ ) rhesus macaques (M. mulatta) obtained from the Oswaldo Cruz Foundation Primate Research Center (Rio de Janeiro, Brazil). Experimental animals were housed indoors in individual steel squeezeback cages in a temperature $\left(25^{\circ} \mathrm{C}\right)$ - and humidity $(60 \pm$ $5 \%$ - controlled environment. Water was provided ad libitum via an automatic watering system, and High Protein Monkey Diet (NUVILAB; Ministério da Agricultura e Reforma Agrária, Brasil), supplemented with eggs, fruits and vegetables, was fed twice daily. The primate facilities are maintained according to the guidelines of the Committee on the Care and Use of Laboratory Animals of the Institute of Laboratory Animal Resources, National Research Council and Health and Human Services (NIH: MD, USA).

The experiments were conducted using a protocol approved by the Institutional Committee of the Center for Biological Evaluation and Care of Research Animals (CEUA-Fiocruz, Protocol \# P0048-00). The monkeys were aclimatized to the laboratory conditions for at least two weeks before the experimental procedures were begun. Monkeys were anaesthetised before infection and prior to each sampling or testing procedure. Animals were initially restrained in their cages, and subsequently they were given, intramuscularly, Ketamine (Ketalar: Ketamine hydrochloride; Parke Davis; $15 \mathrm{mg} / \mathrm{kg}$ body weight) for anesthesia.

Vaccine preparation - The genetically attenuated $\Delta 1$ line of $L$. (L.) major tested here was a non-revertible homozygous $d h f r-t s^{-}(-/-)$mutant organism devoid of drug resistant genes (Gueiros-Filho \& Beverley 1996). Attention was focused to avoid parasite contamination of the $\Delta 1$ line (which was cultivated under controlled conditions and the authenticity of $d h f r-t s^{-}$cultures tested by checking for thymidine auxotrophy). Parasites were also monitored with PCR analysis for DHFR-TS ${ }^{-}$gene [using a 5'-flanking forward primer (SMB389) combined with both SMB390 (a DHFR-TS 3'-flanking) and SMB391 (an internal) reversal primers, which give different DNA sizes] prior to induce infection.

The autoclaved $L$. $(L$.$) major (ALM) vaccine was pre-$ pared as reported (Sharifi et al. 1998), but using the strain LV39 (MRHO/SU/59/P). Freshly suspended BCG produced by Fundação Ataulfo de Paiva (Rio de Janeiro, Brazil) was added to each vaccine vial just before vaccination. The dose of BCG administered corresponded to one-tenth of the standard dose used for vaccination against tuberculosis, equivalent to $5 \times 10^{4}$ colony forming units (CFU). The final formulation contained either $1 \mathrm{mg}$ ALM + this dose of BCG or the same dose of BCG alone.

Vaccination groups and injection - A total of 32 young adult rhesus macaques of either sex were used in this study. Animals were separated into five groups and each group was inoculated as follows: Group A $(n=8)$, injected with attenuated parasites; Group B $(n=8)$, injected with $\mathrm{ALM}+\mathrm{BCG}$; Group C $(\mathrm{n}=6)$, injected with $\mathrm{BCG}$ alone; and Group D $(n=6)$, unvaccinated control (injected with saline). Additionally, four naive monkeys were used as 'normal' controls.

Taking in account previous studies showing either the dose effect of the mutant $L$. $(L$.) major (Titus et al. 1995) or route for administration on the evolution of CL in rhesus monkeys (Amaral et al. 1996, 2001), we used a vaccine consisting of a single dose of $10^{8}$ attenuated parasites, inoculated in above the left eye. The virulence of the $d h f r-t s^{-}$mutant is attenuated when compared to strain LV39 (clone 5 line), requiring about 10-fold more parasites to give similarly rapid progression of the disease in mice (Titus et al. 1995). Following a standardized vaccine protocol used in humans (Momeni et al. 1999), 
the ALM + BCG vaccine was given in three doses (at 30day intervals) in the left deltoid muscle region.

Challenge infection - The strain LV39 (MRHO/SU/ $59 / \mathrm{P}$ ) of $L$. (L.) major was used in the challenge experiments. The strain was typed by serodeme and zymodeme analyses (Grimaldi et al. 1991) in our laboratory before being used for infection. The parasite was maintained by serial subcutaneous passage in hamsters. To obtain suspensions of promastigotes for infection, tissue from chronically infected hamsters was cultured initially in NNN blood agar medium. When promastigotes appeared, the parasites were passaged (no more than three times) into Schneider's Drosophila medium (Gibco), supplemented with $10 \%$ heat-inactivated fetal bovine serum. Parasites were harvested (stationary phase), washed three times using centrifugation in phosphatebuffered saline (PBS), and counted in a Neubauer hemocytometer before use for infection. Primates were each infected in above the right eye by intradermal inoculation of $1 \times 10^{7}$ promastigotes in $0.1 \mathrm{ml}$.

Safety and immunogenic assays - To evaluate the inability of attenuated parasites to cause disease in primates, the infected monkeys were examined every 3 days during 2 weeks following vaccination and then weekly. Parasitological cure was determined using PCR parasite DNA amplification in skin biopsy samples (Pirmez et al. 1999) from inoculation site and/or histopathological analysis.

Analyses of specific immune responses in experimental animals were performed prior to vaccination, every 2-3 weeks following vaccination until week 18 (evaluation of the immunogenicity of vaccine), then at $3,8,13$, and 24 weeks after the challenge infection (evaluation of immunity after vaccination).

DTH reaction to the LST was evaluated as previously described (Amaral et al. 2001). The leishmanin antigen [consisting of pooled heat-killed $L$. (L.) major, $L$. (L.) amazonensis, and L. (Viannia) guyanensis promastigotes suspended in PBS with $0.5 \%$ phenol] was prepared at the Fiocruz (Biomanguinhos Unit), Brazil. A volume $0.1 \mathrm{ml}$ containing $5 \times 10^{6}$ parasites was injected into the left forearm. DTH reaction (skin induration) was measured at the site of injection after $72 \mathrm{~h}$.

The methods followed for peripheral blood leukocyte (PBL) preparations and in vitro lymphocyte blastogenesis assays were those as described (Amaral et al. 1996). The soluble leishmanial (strain LV39) antigens (SLA) for in vitro blast transformation and ELISA assays were prepared as described by Dennis et al. (1986). Briefly, promastigotes were washed and suspended at a final concentration of $10^{9} \mathrm{ml}^{-1}$ in PBS and sonicated at 14-18 amplitude microns for five periods of $45 \mathrm{sec}$ each on ice. The sonicate preparation was centrifuged at $10,000 \mathrm{~g}$ for $30 \mathrm{~min}$. Purified PBL were cultured in the presence of an optimal culture concentration of mitogen (PHA-P at $12.5 \mu \mathrm{g} \mathrm{ml}^{-1}$; Sigma) or SLA (10 $\mu \mathrm{g}$ protein/well). Cultures were incubated at $37^{\circ} \mathrm{C}$ in a humidified atmosphere containing $5 \% \mathrm{CO}_{2}$ for three days in the case of mitogen or for four days in the case of antigens. The cells were pulsed with $\left[{ }^{3} \mathrm{H}\right]$ thymidine (Amersham, Co., U.K.; $1 \mu \mathrm{Ci} /$ well; $5 \mu \mathrm{Ci} / \mathrm{mM}$ ) over the last $18 \mathrm{~h}$ and harvested onto glass fiber filter mats (Titertek,
FlowLab). Radioactive incorporation into DNA was determined by liquid scintillation spectrometry. Results are expressed as the stimulation index (SI, mean cpm stimulated cultures/mean cpm unstimulated cultures). To determine significance, data from the lymphocyte proliferative responses (LPR) of vaccinated and/or challenged monkeys were compared with those of naive animal controls using the unpaired Student's $t$-test.

Cell supernatant IFN- $\gamma$ production by stimulated cells and measurements of IFN- $\gamma$ were determined as described (Amaral et al. 2001). Briefly, purified PBL were adjusted to $2 \times 10^{6}$ cells ml-1 in medium and stimulated with either PHA-P or SLA. Cell culture media were pooled from duplicate wells after $72 \mathrm{~h}$ of stimulation. A rhesus monkey IFN- $\gamma$ ELISA immunoassay kit obtained commercially (Biosource International, Camarillo, CA) was used for the in vitro determination of IFN- $\gamma$ in the supernatants. Streptavidin-peroxidase (HRP) conjugate and stabilized chromogen, tetra-methylbenzidine (TMB) substrate were used for detection, as per the manufacturer's instructions.

Antigen-specific serum antibody (Ab) concentrations were determined by adapting a standard ELISA technique to detect rhesus immunoglobulins (Amaral et al. 2001), using microtiter plate wells coated with SLA, sera of rhesus monkeys (1:50 dilution), and diluted rabbit antimonkey immunoglobulin $\mathrm{G}$ (IgG)-peroxidase (Sigma Chemical Co., St. Louis-MO, USA). The reaction was revealed with biotin-avidin peroxidase system. The substrate consisted of $0.04 \%$ OPD and $0.012 \%$ hydrogen peroxidase in phosphate-citrate buffer, $\mathrm{pH}$ 5. The lower limit of positivity (cut off) was determined by the mean of the negative control +2 s.d. Moreover, the immunoreactivity of serum antibodies to leishmanial (LV39) antigens was determined by Western blot analysis as described (Amaral et al. 1996). The nitrocellulose strips were incubated with sera, then with rabbit anti-monkey IgG-peroxidase conjugate. After rinsing, strips were developed in a satured solution of 3,3'-diaminobenzidine in Tris-HCL buffer containing $0.01 \% \mathrm{H}_{2} \mathrm{O}_{2}$.

Pathology - The size and appearance of leishmanial lesions (lesion development) was followed sequentially in all challenge-infected animals. Lesion area was calculated using the formula $\pi r_{1} r_{2}$, as described (Amaral et al. 1996). Skin biopsies were removed from the border of cutaneous lesions during active stages ( 3 and 6 weeks post challenge) using a 4-mm punch and fixed in 10\% buffered formalin. Paraffin sections were prepared from central and peripheral zones of the lesion and stained with hematoxylin-eosin. For PCR analysis, genomic DNA was extracted from biopsy fragments using an anion-exchange chromatography spin-column following the manufacture's instructions (Pharmacia, Upsalla, Sweden). Final DNA pellet was suspended in $20 \mu \mathrm{l}$ of $10 \mathrm{mM}$ Tris-HCL/1mM EDTA $\mathrm{pH} 8.0$, and stored frozen at $20^{\circ} \mathrm{C}$ until use. A hot-start PCR was performed using oligonucleotides that anneal to the origin of replication of both strands of the minicircle molecules as previously described (Pirmez et al. 1999).

Statistical analysis - When appropriate, Student's unpaired $t$ test was used to determine the statistical significance of the differences between groups. 


\section{RESULTS}

Vaccine safety - The monkeys were vaccinated in groups, as indicated (Fig. 1). The attenuated $L$. (L.) major vaccine dose was given as a single $0.1 \mathrm{ml}$ s.c. injection (containing $10^{8}$ stationary phase promastigotes) in above the left eye on day 0 . The ALM + BCG vaccine was given in three doses administered at 30-day intervals, while the other groups (treated similarly with either BCG alone or saline) served as controls.

Using PCR assay follow-ups (data not shown), we monitored the establishment and resolution of infection caused by attenuated parasites. The results indicate that the infection persisted in all attenuated parasite-vaccinated animals for up to 2-3 months, nonetheless they were incapable of causing disease in susceptible monkeys. Leishmania DNA was not detected at the site of inoculation by 18 weeks following vaccination. The histopathological findings at this time point of infection showed a non-specific focal mononuclear cell infiltrate in the dermis, which included small lymphocytes clustered around post capillary venules of the vascular plexus (Fig. 5E).

There was no severe post-vaccination reaction in the two experimental groups that had received BCG, such as those (induration, ulceration, or secretions at the injection site) usually associated with BCG vaccination in humans (Armijos et al. 1998, Momeni et al. 1999). Side-effects due to the local inflammatory reaction (such as redness and swelling), mostly confined to the initial three days, were more marked and frequent in the ALM + BCG-vaccinated group as compared to the controls.

Immunogenicity - The DTH reaction to LST was used as in vivo correlates of cellular immunity. As illustrated (Fig. 1), abortive infection with the attenuated $L$. (L.) major and/or periodic boosting in vaccinated monkeys using inactivated parasites did not promote LST conversion, but DTH responses developed in all challenge-infected animals. However, the LST induration size values (mean \pm SE) were not significantly different $(p>0.05)$ in vaccine groups when compared with controls. In contrast, both

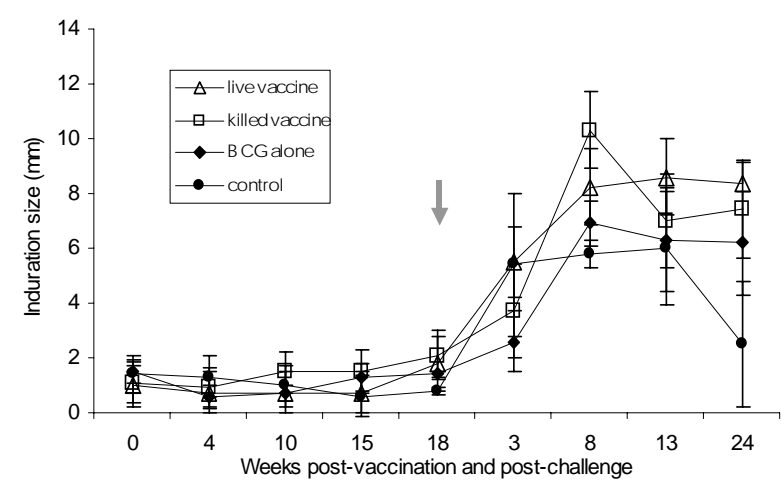

Fig. 1: leishmanin skin test responses were measured to assess levels of cell-mediated immunity in vivo developed in experimental rhesus macaques following vaccination and/or challenge infection (at the time point indicated by arrow) with Leishmania (L.) major. Animals were measured at $72 \mathrm{~h}$ after injection of $0.1 \mathrm{ml}$ containing $5 \times 10^{6}$ heat-killed promastigotes into the shaven area of the right forearm and results are expressed as the diameter of skin induration in millimeters. Each point represents mean \pm SE of 8 (vaccinees) or 6 (controls) monkeys.
L. (L.) major vaccines resulted in the stimulation of a parasite-specific lymphoproliferative response in a high percentage of vaccinated monkeys (Fig. 2). It was found a very high degree of variability in the level of T-cell responsiveness by individual over time, but a mean positivity rate of $93 \%$ was detected by 10 weeks following vaccination. The overall lymphocyte blastogenesis positivity (measured at $4,10,15$ and 18 weeks postvaccination) in attenuated parasite-vaccinated monkeys was $79 \%$ (versus $75 \%$ in ALM + BCG-vaccinated animals), while the positivity rates measured at 18 weeks postvaccination were lower in both vaccine groups $(67 \%$ and $60 \%$, respectively). Following challenge, groups receiving vaccine had higher but similar levels of in vitro lymphocyte response, except at one time point ( 8 weeks p.i.) when attenuated $L$. (L.) major-vaccinated monkeys had significantly $(\mathrm{P}<0.05)$ higher SI values as compared with the saline group.

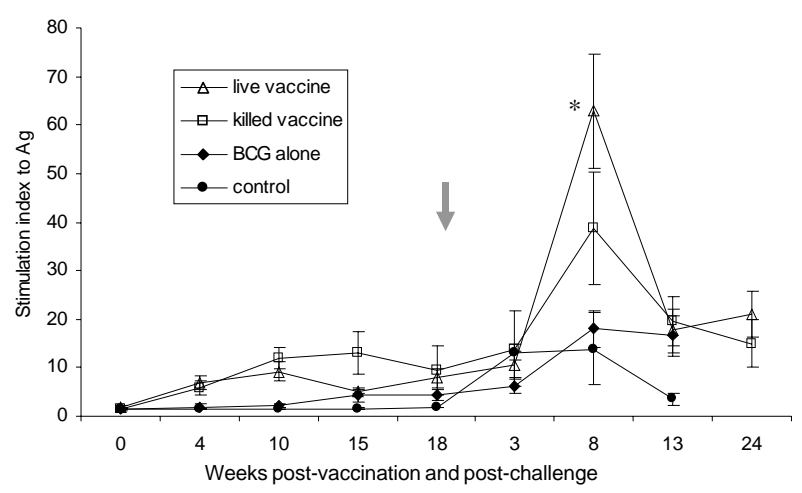

Fig. 2: proliferative responses in vitro to soluble leishmanial antigen of peripheral blood leukocytes from experimental rhesus monkeys prior to and following vaccination and challenge infection. Results are expressed as the stimulation index (SI, mean cpm stimulated cultures/mean cpm unstimulated cultures). Each point represents mean \pm SE of 8 (vaccinees) or 6 (controls) monkeys. The animals were challenged with virulent Leishmania (L.) major on week 18 post-vaccination, as indicated (arrow). *Significant difference (p < 0.05) compared with the saline (control) group.

Following vaccination, however, no evidence of Agspecific IFN- $\gamma$ response was detected in monkeys from either experimental group when compared with healthy monkeys (data not shown). In fact only three animals from each vaccine group, after challenge, produced IFN- $\gamma(40-$ $80 \mathrm{pg} / \mathrm{ml}$ ) above the level found at time zero or in healthy control monkeys (10-15 pg/ml).

The serum levels of specific antibodies against SLA were assessed by ELISA at different time points following vaccination and/or challenge infection (Fig. 3). All experimental groups developed at least a 2-fold increase in Ag-specific IgG titer by 10 weeks post-vaccination, while the saline group failed to develop significant antibodies before challenge. Three weeks following challenge, all groups had higher levels of specific anti-L. (L.) major antibodies, but $\mathrm{IgG}$ responses in vaccinated monkeys were not above those of the controls. The amount of Agspecific IgG antibodies continued to increase during active infection (peaking at 8 weeks p.i.), after which lev- 
els declined in animals with healing lesion. Western blots analyses of SLA were performed employing immune sera from monkeys at various times post-vaccination and/or after challenge (data not shown). The IgG antibodies produced by $L$. (L.) major-challenged animals bound to multiple components (bands ranging from 35 to $210 \mathrm{kDA}$ ), most notably a Leishmania antigen of $35 \mathrm{kDA}$, which was also recognized by sera from vaccinated animal groups collected prior to challenge infection.

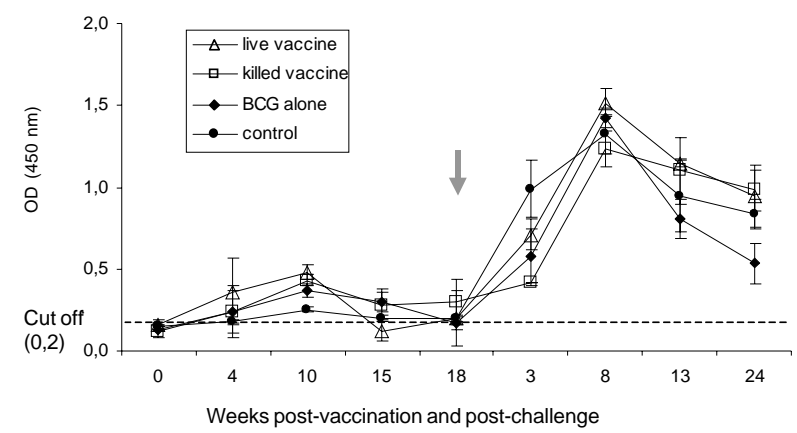

Fig. 3: changes in serum levels of Leishmania-specific IgG antibodies in rhesus macaques detected among vaccine and control groups. Each point represents the optical density value (mean $\pm \mathrm{SE}$ ) at 450 $\mathrm{nm}\left(\mathrm{OD}_{450}\right)$ of sera from 8 (vaccinees) or 6 (controls) monkeys tested (1:50 dilution), as determined by enzyme-linked immunosorbent assay. Experimental animals were challenged with virulent Leishmania (L.) major at the time point as indicated by arrow.

Efficacy - The poor response to vaccination (using either attenuated or killed vaccine) obtained was evident when lesion development was followed over time after challenge infection with virulent $L$. (L.) major. As illustrated (Fig. 4), all of the animals developed CL at the site of infection (Fig. 5A-D). The time of skin lesion onset and healing was similar in infected monkeys from either control or experimental groups. Although vaccinated animals (in particular those receiving ALM + BCG) showed accentuated disease (Fig. 5B), the mean lesion size at different time points of infection in vaccine groups was not significantly different from the control groups (Fig.

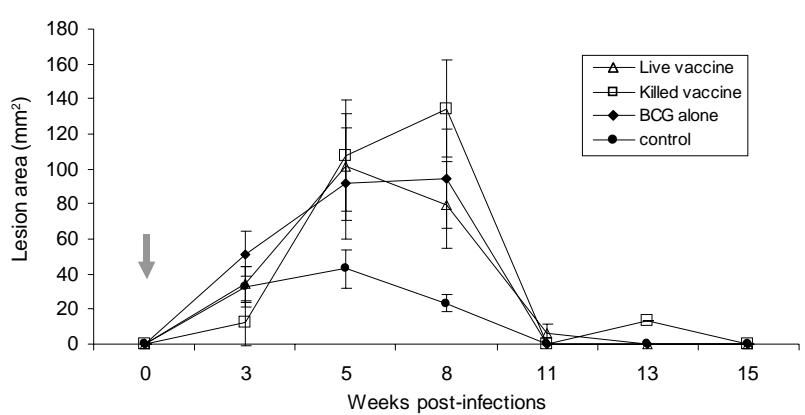

Fig. 4: time course of skin lesion development in Macaca mulatta monkeys injected with either attenuated or killed Leishmania vaccine, and in control monkeys, after challenge with $10^{7} \mathrm{~L}$. $(\mathrm{L}$.) major virulent promastigotes. The animals were inoculated intradermally on the upper eyelid at weeks 18 post-vaccination (arrow indicates start of the challenge infection). Lesions were scored by calculating the total lesion area as indicated (see Materials and Methods). Data are mean \pm SE of 8 (vaccinees) or 6 (controls) monkeys.
4). A typical ulcerating nodular CL lesion progressed rapidly (peaking at 5 to 8 wks p.i.) and subsequently regressed and healed (most of the skin lesions had disapeared from infected animals by $11 \mathrm{wks}$ p.i.).

Distinct histopathological patterns were observed during lesion development in $L$. (L.) major-infected rhesus monkeys, but similar pathologic features were found at skin lesion biopsies obtained among control and vaccinated animal groups. In early phases ( 3 weeks p.i.) of developing ulcerated skin lesions, a non-specific chronic mononuclear infiltrate predominate in the dermis, which evolved to the formation of tuberculoid-type granulomatous nodules (Fig. 5F), then representing the principal feature in late stages ( 8 weeks p.i.).

\section{DISCUSSION}

The enhanced protective immunity following selfhealing CL is attributed to the development of a strong Leishmania-specific $\mathrm{CD} 4^{+} \mathrm{Th} 1$ cell response (Kemp et al. 1994). Although results from animal studies can not be directly extrapolated to human disease, the use of appropriate animal models in evaluating the efficacy of candidate vaccines can be very informative. Current research employing rodent (Gurunathan et al. 1998) and primate (Kenney et al. 1999, Campos-Neto et al. 2001, Gicheru et al. 2001) models is providing the foundation for studies designed to defining vaccine requirements (such as candidate antigens and adjuvants or delivery systems) for sustained cellular immunity to Leishmania infection.

The only prophylactic vaccination strategy so far employed in humans with proved success against CL has required prior controlled induction of disease with a virulent parasite (Naggan et al. 1972). Both cellular and humoral immune response to leishmanial antigens (Green et al. 1983) and resistance to reinfection usually developed in vaccinees (Naggan et al. 1972). Clinical and field studies indicate that nonliving Leishmania vaccines are apparently safe and possess immunogenic properties, but their protective efficacy is unclear (reviewed by Grimaldi 1995). Positive DTH response to the LST developed in vaccinees, which seems to increase the recipient 's chance of being protected (Armijos et al. 1998). However, whether nonliving Leishmania can promote similar levels of immunity in humans as virulent Leishmania is at present unknown.

Problems associated with the use of a virulent vaccine could be avoided using recombinant attenuated Leishmania. The successful immunization of susceptible strains of mice (Titus et al. 1995) with $L$. (L.) major mutants lacking the dihydrofolate reductase-thymidylate synthetase (DHFR-TS) by gene replacement (GueirosFilho \& Beverley 1996) has established an approach using rationally-designed safe avirulent vaccines in Leishmania vacinology. The ability of $d h f r-t s^{-}$parasites to invade macrophages and persist briefly in the animal host for up to three months [as shown in both rodent (Titus et al. 1995) and primate models (this study)] may reflect a locus-specific advantage of genetically attenuated parasites in prolonging the period and diversity of antigen delivery by Leishmania. Considering the potential appli- 
cation of this vaccination approach to humans, the first question that we addressed was whether primates abortively infected with the mutant parasite would be protected against subsequent challenge with virulent $L$. (L.) major. Our rationale to have comparatively tested the ALM $+\mathrm{BCG}$ vaccine was based on the current question whether inactivated parasites would promote similar levels of protection in monkeys as living parasites.
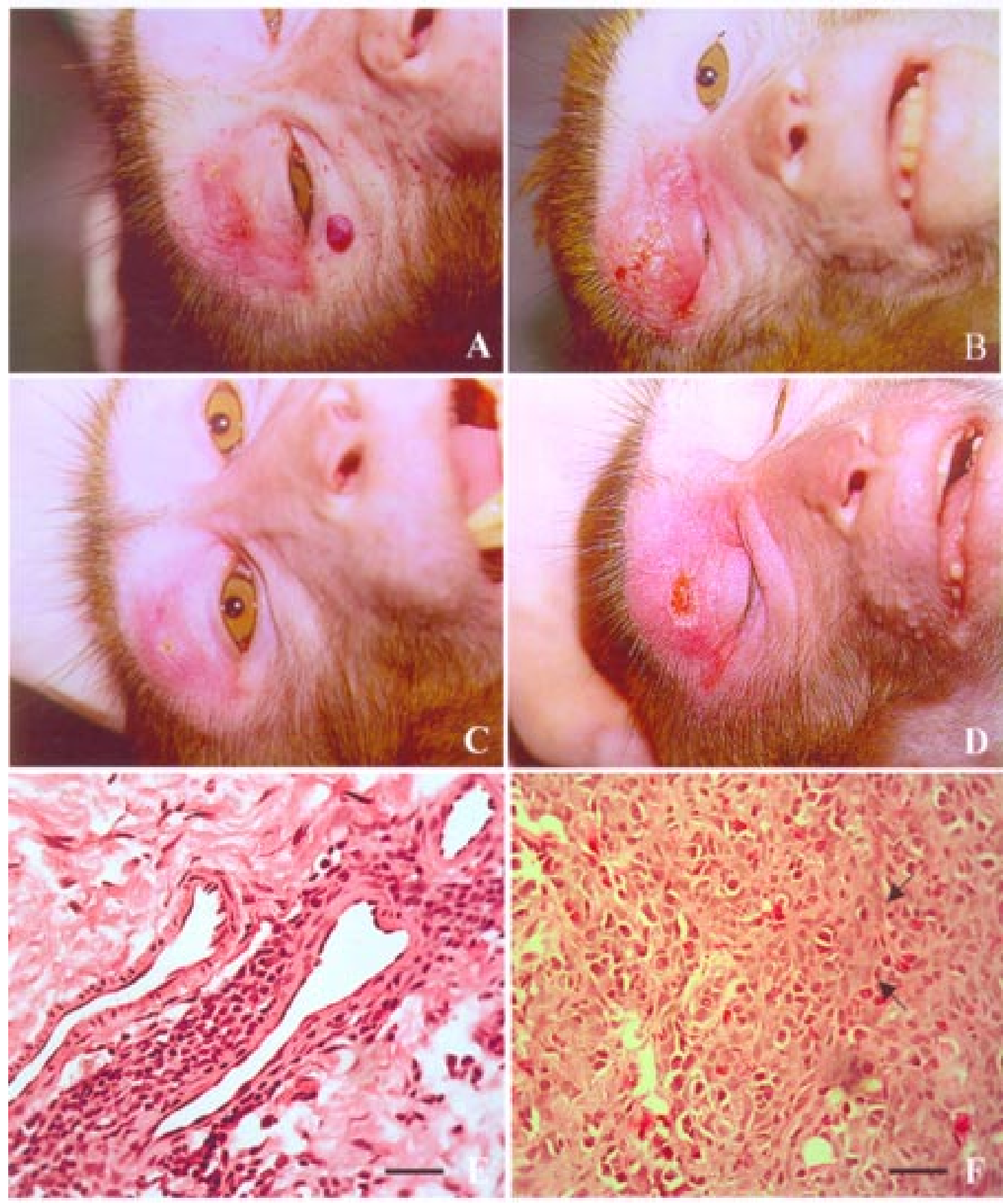

Fig. 5: self-healing cutaneous leishmaniasis in rhesus macaques following challenge with Leishmania (L.) major. Shown are pictures of skin lesion development at 8 week p.i. among experimental groups. A: monkey vaccinated with attenuated, live parasites; B: monkey vaccinated with ALM + BCG; C: monkey immunized with BCG alone; and D: monkey unvaccinated control (injected with saline). Also shown are microscopic characteristics of the inflammatory reactions in a monkey injected with attenuated parasites, as detected by 18 weeks following vaccination (E) and in a control animal challenged with virulent $L$. (L.) major (F). Note in this section (F) from a developing skin lesion (D), a mononuclear cell infiltrate containing differenciated macrophages (arrows) in the dermis, typical of a cellmediated immune response-induced granuloma reaction. Hematoxylin and eosin stain; bar $=50 \mu \mathrm{m}$. 
et al. 1996, 2001), both $L$. (L.) amazonensis- and $L$. (L.) major-infected rhesus monkeys that had recovered from skin lesions developed clinical resistance to homologous challenge, indicating that acquired protective immunity against the parasite occurred. Moreover, excellent protective effects of vaccination of rhesus monkeys have been obtained when a mixture of IL-12 and alum is combined with either heat-killed Leishmania promastigotas (Kenney et al. 1999) or recombinant parasite antigens (CamposNeto et al. 2001).

A likely explanation for the failure in promoting immunity is that none of the immunizing regimens used in this study stimulated a Th1 response and, as a consequence, primates could not control the subsequent challenge. In rodent models, protective immunity is dependent on the ability to mount an IL-12-driven CD4 ${ }^{+}$ type 1 helper T-cell (Th1) response (Reed \& Scott 2000). Unlike as it occurs with proven protective immunity in self-healing CL (Kemp et al. 1994), in the present study little or no IFN- $\gamma$ was detected in tested animals, which may indicate a limit of the model. Nevertheless, it is very possible that this could in fact represent a false-negative result, either because the time point to measure the cytokine was not the optimal one, or because the kit was not sensitive enough to detect its production.

Studies with the vervet monkey model for CL have demonstrated that resistance to the challenge in this animal system is correlated with a Th1-like activity response as revealed by an increased production of IFN- $\gamma$ by the responding T cells and strong DTH responses (Olobo et al. 1992). However, more recent data suggest that protection against CL in either vaccinated rhesus macaques (Kenney et al. 1999) or vervets (Gicheru et al. 2001) may require more than the activation of Leishmaniaspecific IFN- $\gamma$-producing T cells. Of special interest are more recent data from vaccine trials in the rhesus monkey model combining recombinant Leishmania antigens with IL-12 and alum as adjuvants (Campos-Neto et al. 2001). In those experiments, the immunizing regimen was fully effective, but neither augmented Ag-specific IFN- $\gamma$ response nor DTH reaction to LST was a good predictive parameter of protection. These data have important implications for designing or evaluating a vaccine against the disease.

The relative variability in level of protection induced by vaccination against $C L$ is probably due to several factors influencing the nature of immunity attained. It has been shown that protective immunity induced in mice following vaccination is variable according to the size or number of immunizing dose, or route of immunization (Liew et al. 1985, Menon \& Bretscher 1996). Therefore, success of a vaccine depends on the adoption of a suitable vaccination protocol. Infection of mice with a chemically mutagenized avirulent form of $L$. (L.) major conferred immunity when the route of injection was either intravenous or intraperitoneal (McGurn et al. 1990). Perhaps had a second dose (Veras et al. 1999), or another route for immunization been tested as in mice intravenously (Titus et al. 1995), we would have seen better results employing $d h f r-t s^{-} L$. (L.) major as safe live vaccine. Our rationale for not have tested the attenuated vaccine via intravenous injection was due to potential problems that would be associated using this vaccine protocol in humans. Another factor that can influence vaccine-induced protection is the time between the last vaccination and challenge infection. In the murine studies, animals were challenged one week after vaccination (Titus et al. 1995, Veras et al. 1999), while in the present study monkeys were not challenged until four months after the primary infection with attenuated parasites. However, at the same time intervals, the abortive infection persisted, as well as specific proliferative responses were detected in a proportion of attenuated parasite-vaccinated monkeys $(67 \%)$.

Whether the intensity of duration of the elicited responses and their roles in ALM-plus-BCG vaccineinduced immunity may vary according to the nature or dose of the BCG used as adjuvant, or the genetic variability of the host remains to be established in primate models. The protective potential of killed $L$. $(L$. ) major along with BCG was also evaluated against $L$. ( $L$. donovani in Indian langur monkeys (Dube et al. 1998). All challenge-infected primates developed infection, but effective protection was apparently observed in monkeys receiving a triple dose vaccination (each of $1 \mathrm{mg}$ ALM plus $1 \mathrm{mg}$ BCG). In our experiments the dose of BCG used was much lower, but several consistent patterns of sensitivity development could be discerned in healthy rhesus macaques that had received $3.9 \times 10^{5} \mathrm{CFU}$ of $\mathrm{BCG}$ vaccine, as described (Chaparas et al. 1975).

With respect to humoral response, a 2-fold increase in $\mathrm{Ag}$-specific IgG titer was seen in all experimental groups, but only by 10 weeks post-vaccination. These data are similar with those obtained in field studies (Sarples et al. 1994) showing a pattern of anti-leishmanial antibody titers consistent with a response to the skin test antigen.

For evaluating the safety of both vaccine protocols, we have examined all tested monkeys for side effects over time following vaccination and challenge infection. Injection of either one dose attenuated parasites or three doses ALM with BCG, or BCG alone used in this study was not associated with appreciable skin alteration at the site of injection, but vaccination sometimes resulted in exacerbation of the skin lesion following challenge. Given that none of the monkeys injected with attenuated $L$. $(L$.) major developed disease, potentially $d h f r-t s^{-}$mutant parasites could be used as a delivery system for other antigen and/or adjuvant (e.g., IL-12), which would elicit substantial Th1-like response in vaccinees. Taken together, these observations show that the rhesus model can be effective for evaluating safety, immunogenicity, and efficacy of candidate vaccines against leishmanial infection.

\section{ACKNOWLEDGMENTS}

To Dr Antonio da Mota Marinho and the staff in the Fiocruz Animal Care Facility for their assistance with daily care and procedures on the macaques.

\section{REFERENCES}

Amaral VF, Ransatto VAO, Conceição-Silva F, Molinaro E, Ferreira V, Coutinho SG, McMahon-Pratt D, Grimaldi Jr G 1996. The Asian Rhesus macaques (Macaca mulatta) as an experimental model for study of cutaneous leishmaniasis. 
Exp Parasitol 82: 34-44.

Amaral VF, Teva A, Porrozzi R, Silva AJ, Pereira MS, OliveiraNeto MP, Grimaldi Jr G 2001. Leishmania (Leishmania) major-infected rhesus macaques (Macaca mulatta) develop varying levels of resistance against homologous reinfections. Mem Inst Oswaldo Cruz 96: 795-804.

Antunes CM, Mayrink W, Magalhaes PA, Costa CA, Melo MN, Dias M, Michalick MS, Williams P, Lima AO, Vieira JB, Schettini APM 1986. Controlled field trials of a vaccine against New World cutaneous leishmaniasis. Int J Epidemiol 15: $572-580$.

Armijos RX, Weigel MM, Aviles H, Maldonado R, Racines J 1998. Field trial of a vaccine against New World cutaneous leishmaniasis in an at-risk child population: safety, immunogenicity, and efficacy during the first 12 months of follow-up. J Infect Dis 177: 1352-1357.

Campos-Neto A, Porrozzi R, Greeson K, Coler RN, Webb JR, Seiky YA, Reed SG, Grimaldi Jr G 2001. Protection against cutaneous leishmaniasis induced by recombinant antigens in murine and nonhuman primate models of the human disease. Infect Immun 69: 4103-4108.

Chaparas SD, Good RC, Janicki BW 1975.Tuberculin-induced lymphocyte transformation and skin reactivity in monkeys vaccinated or not vaccinated with Bacille Calmette-Guérin, then challenged with virulent Mycobacterium tuberculosis. Am Rev Resp Dis 112: 43-47.

Dennis VA, Lujan R, Chapman WL Jr, Hanson WL 1986. Leishmania donovani: cellular and humoral immune responses after primary and challenge infections in squirrel monkeys, Saimiri sciureus. Exp Parasitol 61: 319-334.

Dube A, Sharma P, Srivastava JK, Misra A, Naik S, Katiyar JC 1998. Vaccination of langur monkeys (Presbytis entellus) against Leishmania donovani with autoclaved L. major plus BCG. Parasitology 116: 219-221.

Dye C 1996. The logic of visceral leishmaniasis control. Am J Trop Med Hyg 55: 125-130.

Gicheru MM, Olobo JO, Anjili CO, Orago AS, Modabber F, Scott P 2001. Vervet monkeys vaccinated with killed Leishmania major parasites and interleukin-12 develop a type 1 immune response but are not protected against challenge infection. Infect Immun 69: 245-251.

Green MS, Kark J D, Witzum E, Greenblat CL, Spira DT 1983. Frozen stored $L$. tropica vaccine: the effect of dose, route for administration and storage on the evolution of clinical lesion. Two field trials in the Israel defense forces. Trans $R$ Soc Trop Med Hyg 77: 152-159.

Grimaldi Jr G 1995. Meeting on vaccine studies towards the control of leishmaniasis. Mem Inst Oswaldo Cruz 90: 553556.

Grimaldi Jr G, Tesh RB 1993. Leishmaniasis of the New World: current concepts and implications for future research. Clin Microb Rev 6: 230-250.

Grimaldi Jr G, Momen H, Naiff RD, McMahon-Pratt D, Barrett TV 1991. Characterization and classification of leishmanial parasites from humans, wild animals, and sand flies in the Amazon region of Brazil. Am J Trop Med Hyg 44: 645-661.

Gueiros-Filho FJ, Beverley SM 1996. Selection against Dihydrofolate Reductase-Thymidylate Synthase (DHFR$T S)$ as a probe of genetic alterations in Leishmania. Molec Cell Biol 16: 5655-5663.

Guirges SY 1971. Natural and experimental re-infection of man with Oriental sore. Ann Trop Med Parasitol 65: 197-205.

Gurunathan S, Prussin C, Sacks DL, Seder RA 1998. Vaccine requirements for sustained cellular immunity to an intracellular parasitic infection. Nat Med 4: 1409-1415.

Kemp M, Hey AS, Kurtzhals JÁ, Christensen CB 1994. Dichotomy of the human $\mathrm{T}$ cell response to Leishmania antigens. I. Th1-like activity response to Leishmania major promastigote antigens in individuals recovered from cutaneous leishmaniasis. Clin Exp Immunol 96: 410-415.

Kenney RT, Sacks DL, Sypek JP, Vilela L, Gam AA, EvansDavis K 1999. Protective immunity using recombinant human IL-12 and alum as adjuvants in a primate model of cutaneous leishmaniasis. J Immunol 163: 4481-4488.

Liew FY, Hale C, Howard JG 1985. Prophylactic immunization against experimental leishmaniasis. IV. Subcutaneous immunization prevents the induction of protective immunity against fatal Leishmania major infection. J Immunol 135: 2095-2101.

Mayrink W, da Costa CA, Magalhães PA, Melo MN, Dias M, Oliveira Lima A, Michalick MS, Williams P 1979. A field trial of a vaccine against American dermal leishmaniasis. Trans $R$ Soc Trop Med Hyg 73: 385-387.

McGurn M, Boon T, Louis JÁ. Titus RG 1990. Leishmania major: nature of immunity induced by immunization with a mutagenized avirulent clone of the parasite in mice. Exp Parasitol 71: 81-89.

Menon JN, Bretscher PA 1996. Characterization of the immunological memory state generated in mice susceptible to Leishmania major following exposure to low doses of L. major and resulting in resistance to a normally pathogenic challenge. Eur J Immunol 26: 243-249.

Momeni AZ, Jalayer T, Emamjomeh M, Khamesipour A, Zicker F, Ghassemi RL, Dowlati Y, Sharifi I, Aminjavaheri M, Shafiei A, Alimohammadian MH, Hashemi-Fesharki R, Nasseri K, Godal T, Smith PG, Modabber F 1999. A randomised, double-blind, controlled trial of a killed L. major vaccine plus BCG against zoonotic cutaneous leishmaniasis in Iran. Vaccine 17: 466-472.

Naggan L, Gunders AE, Michaeli D 1972. Follow-up study of a vaccination programme against cutaneous leishmaniasis. II. Vaccination with a recently isolated strain of $L$. tropica from Jericho. Trans $R$ Soc Trop Med Hyg 66: 239-243.

Olobo JO, Reid GD, Githure JI, Anjili CO 1992. IFN-gamma and delayed-type hypersensitivity are associated with cutaneous leishmaniasis in vervet monkeys following secondary rechallenge with Leishmania major. Scand $J$ Immunol (Suppl.) 11: 48-52.

Pirmez C, Trajano V, Oliveira-Neto MP, da-Cruz AM, Gonçalves-da-Costa SC, Catanho M, Degrave W, Fernandes O 1999. Use of PCR in diagnosis of human American tegumentary leishmaniasis in Rio de Janeiro, Brazil. J Clin Microbiol 37: 1819-1823.

Reed SG, Scott P 2000. Immunologic mechanisms in Leishmania. In MW Cunningham, RS Fujinami (eds), Effects of Microbes on the Immune System, Lippincott Williams, Wilkins, Philadelphia, p. 537-554.

Sarples CE, Shaw MA, Castes M, Convit J, Blackwell JM 1994. Immune response in healthy volunteers vaccinated with BCG plus killed leishmanial promastigotes: antibody responses to mycobacterial and leishmanial antigens. Vaccine 12: 1402-1412.

Sharifi I, FeKri AR, Aflatonian MR, Khamesipour A, Nadim A, Mousavi MR, Momeni AZ, Dowlati Y, Godal T, Zicker F, Smith PG, Modabber F 1998. Randomised vaccine trial of a single dose of killed Leishmania major plus BCG against anthroponotic cutaneous leishmaniasis in Bam, Iran. Lancet 351: $1540-1543$.

Titus RG, Gueiros-Filho FJ, de Freitas LAR, Beverley SM 1995. Development of a safe live Leishmania vaccine line by gene replacement. Proc Natl Acad Sci USA 92: 10267-10271.

Veras PST, Brodskyn CI, Balestieri FMP, Freitas LD, Ramos A, Queiroz A, Barral A, Beverley SM, Barral-Neto M 1999. A dhfr-ts- Leishmania major knockout mutant crossprotects against Leishmania amazonensis. Mem Inst Oswaldo Cruz 94: 491-496. 